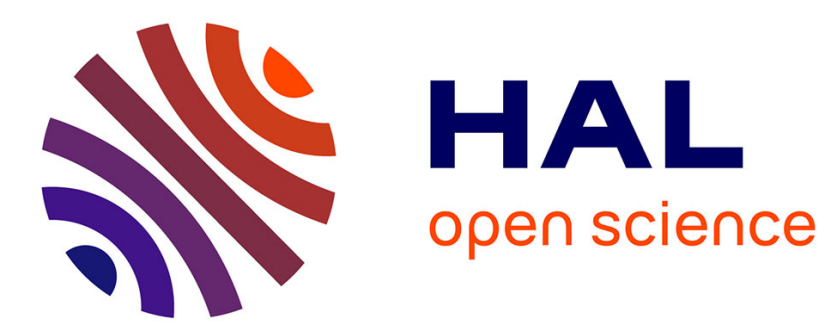

\title{
Impact on multi-layered polypropylene foams
}

Laurent Maheo, Philippe Viot

\section{To cite this version:}

Laurent Maheo, Philippe Viot. Impact on multi-layered polypropylene foams. International Journal of Impact Engineering, 2013, 53, pp.84-93. 10.1016/j.ijimpeng.2012.03.011 . hal-01009901

\section{HAL Id: hal-01009901 https://hal.science/hal-01009901}

Submitted on 18 Jun 2014

HAL is a multi-disciplinary open access archive for the deposit and dissemination of scientific research documents, whether they are published or not. The documents may come from teaching and research institutions in France or abroad, or from public or private research centers.
L'archive ouverte pluridisciplinaire HAL, est destinée au dépôt et à la diffusion de documents scientifiques de niveau recherche, publiés ou non, émanant des établissements d'enseignement et de recherche français ou étrangers, des laboratoires publics ou privés. 


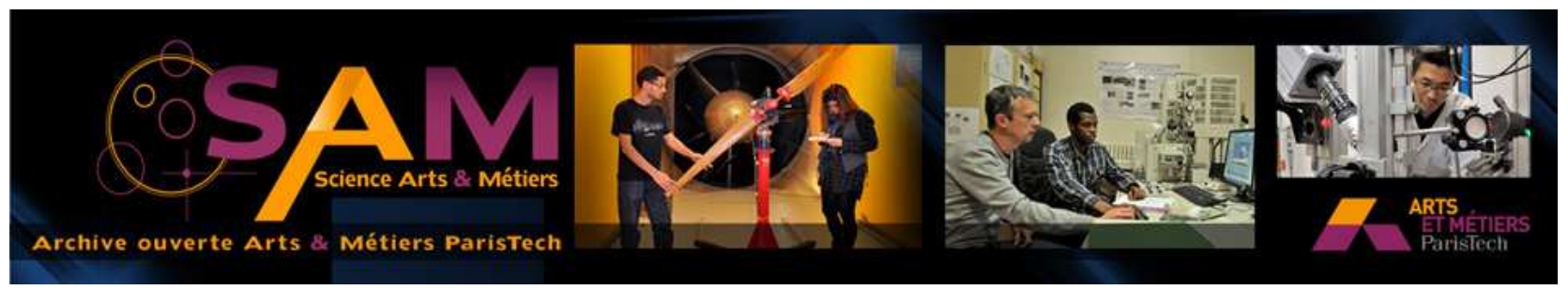

Science Arts \& Métiers (SAM)

is an open access repository that collects the work of Arts et Métiers ParisTech researchers and makes it freely available over the web where possible.

This is an author-deposited version published in: http://sam.ensam.eu Handle ID: .http://hdl.handle.net/10985/8271

\section{To cite this version :}

Laurent MAHEO, Philippe VIOT - Impact on multi-layered polypropylene foams - International Journal of Impact Engineering - Vol. 53, p.84-93 - 2013 


\title{
Impact on multi-layered polypropylene foams
}

\author{
L. Maheo ${ }^{\mathrm{a}, \mathrm{b}, \mathrm{c}}, \mathrm{P}$. Viot ${ }^{\mathrm{a}, \mathrm{b}, \mathrm{c}, *}$ \\ ${ }^{a}$ Arts et Metiers ParisTech, I2M-DuMAS, UMR 5295, F-33400 Talence, France \\ ${ }^{\mathrm{b}}$ CNRS, I2M-DuMAS, UMR 5295, F-33400 Talence, France \\ ${ }^{\mathrm{c}}$ University of Bordeaux, I2M-DuMAS, UMR 5295, F-33400 Talence, France
}

Keywords:

Foams

Multi-scale

Dynamic compression

Numerical simulation

Experiments

\begin{abstract}
A B S T R A C T
Foams, and particularly the polypropylene foam, are more and more often used in the area of injury protection and passive safety for its energy absorption capacity. This multi-scale material is constituted of mesoscopic beads with a large variability of the material properties. To study the effects of these mesoscopic heterogeneities on both the macroscopic and the local behaviors, numerical simulations on virtual volumes of foam under dynamic loading have been performed. The influence of the organized system of heterogeneities has also been studied in the cases of a random distribution and a multi-layered volume. Experimental dynamic compressive tests have been performed on multi-layered volumes of foam and compared with the results of the Finite Element Method.
\end{abstract}

\section{Introduction}

Foams are more and more often used as a cushioning structure (e.g. Helmets) in order to protect against impacts [1]. Following a first elastic stage, a great amount of energy of the impact can be dissipated by foams during the stress plateau [2]. Recently, energy absorption capacity of foams has been enhanced by using layered [3] or functionally graded [4-6] foams. Several studies have been reported on different kinds of expanded materials such as polyurethane [1], polystyrene [3-5] or syntactic epoxy [6]. Authors of previous studies showed special interest in the use of foams in the area of injury protection and passive safety.

The expanded polypropylene (EPP) material of this study is constituted of large mesoscopic beads and microscopic cells [7]. This kind of material shows a multi-scale structure where the millimetric beads (about $2-5 \mathrm{~mm}$ ) are themselves constituted of microscopic closed cells (Fig. 1a). Three scales can therefore be defined: the macroscopic scale for the foam sample, the mesoscopic scale for the beads and the microscopic scale for the microscopic cells. These microscopic and mesoscopic structures have an influence on the macroscopic behavior, as shown experimentally by Viot et al. [8] and Bouix et al. [9]. Several models have

\footnotetext{
* Corresponding author. Arts et Metiers ParisTech, I2M-DuMAS, UMR 5295, F-33400 Talence, France. Tel.: +33 556845 362; fax: +33 556845366.

E-mail address: philippe.viot@ensam.eu (P. Viot).
}

already been implemented in many software calculation codes to represent the foam behavior. In spite of its limits to represent strain localizations, the Finite Element (FE) Method remains the most used one. Most of foam models are proposed to FE code users in order to numerically estimate the macroscopic foam structure response under static and dynamic loadings. Using the FE method, the strain localizations can therefore be approached by introducing some variability of the material properties in the numerical sample. In this case, the variability applied to the numerical sample of foam can be obtained from X-Ray micro-tomography and a study of image analysis. Voxel-based datasets can therefore define a voxelbased FE mesh [10]. Another way to avoid this heavy and costly technique is to numerically build a virtual sample [5].

In the present study, authors proposed to show the effects of the mesoscopic heterogeneities at the scale of the beads on both the macroscopic and the local behaviors with a numerical approach using virtual samples of foam. The influence of the organized system of heterogeneities has also been studied in the case of a random distribution or a multi-layered volume.

Experimental investigations were undertaken to characterize the behavior of the foam loaded to uni-axial compressive impacts. FE simulations of such loadings have been performed on virtual samples of foam including heterogeneous beads of variable properties. The material properties of this foam are obtained from the complete experimental study of Bouix et al. [9] which gives the macroscopic behavior of the EPP material for several values of density and several values of strain rate. 

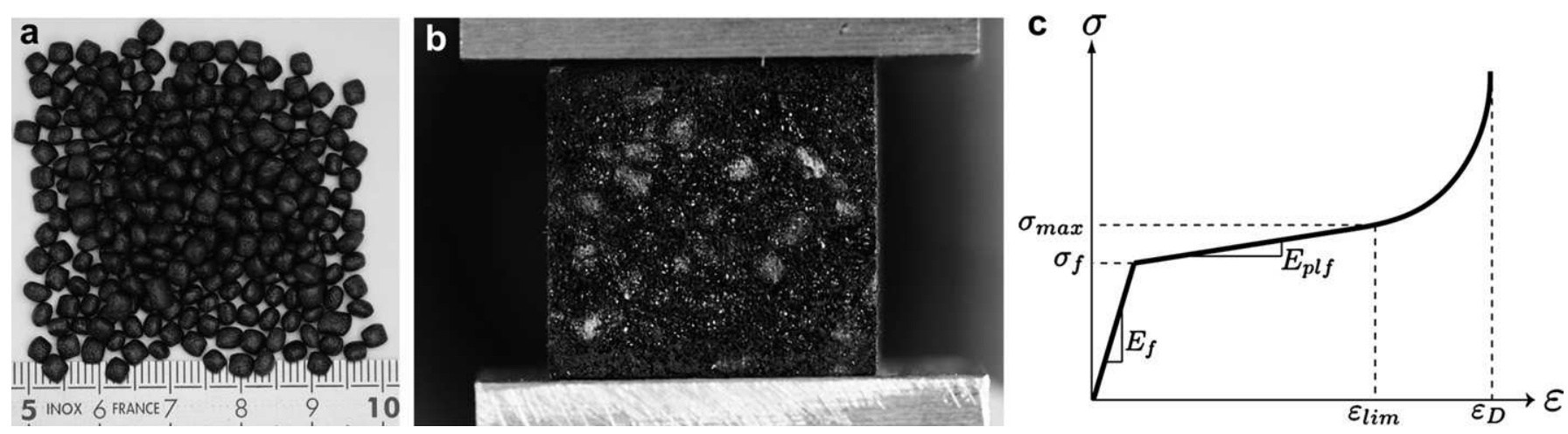

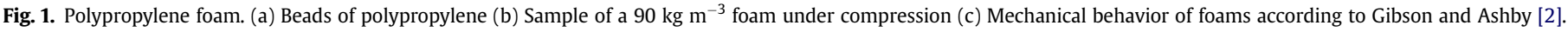

The first part of the work (i.e. Section 2) consisted in studying some virtual samples of foam in order to determine numerically if the presence of heterogeneities, i.e. the mesoscopic beads, can modify the macroscopic and the mesoscopic behaviors. The second part (i.e. Section 3) deals with the capacity of multi-layered foams to absorb the impact energy. Three kinds of these foams have been tested experimentally and numerically and show their energy absorption capacities in such impact loadings. Finally, the results of this article are summarized in Section 4.

\section{Material}

\subsection{Experimental compressive tests}

The foam used in this study is an expanded polypropylene (EPP) material used most of time in the manufacturing process of sport helmets. During processing, the expanded plastic foam beads (Fig. 1a) are injected into a mold where individual beads are fused together under steam heat and pressure to form a medium without inter-bead porosity. The EPP material presented (Fig. 1b) is constituted of millimetric porous beads. These beads are themselves constituted of microscopic cells. The behavior of this foam under compression (Fig. 1c) presents three distinct regimes [2]: an elastic behavior followed by a stress plateau (where the stress is nearly constant for a large range of strain) and finally a densification step.

An important experimental work conducted by Bouix et al. [9] enabled us to obtain a large dataset of stress-strain curves for different values of density (34-150 $\mathrm{kg} \mathrm{m}^{-3}$ ) and for a large range of strain rates. A Zwick electromechanical testing machine was used for quasistatic compression tests where the strain rate was about to $10^{-1} \mathrm{~s}^{-1}$. Dynamic compressive tests were performed with the well-known Split Hopkinson Pressure Bars to reach strain rates of
$10^{3} \mathrm{~s}^{-1}$. The experiments were completed using a Flywheel device for the intermediate strain rates $[9,11]$.

Another experimental work by Viot et al. [7] based on the X-Ray micro-tomography technique studied the microstructure of the EPP material. Authors analyzed micro-tomography images and observed a strong heterogeneity at the bead scale. The value of bead's density can be evaluated from 10 to $200 \mathrm{~kg} \mathrm{~m}^{-3}$. However this study could not be used to determine bead densities with accuracy because a significant error on local porosity is generated by measurements. For our study, the EPP material is different from the one used by Viot et al. [7] and the density range used in the FE simulation is therefore chosen arbitrarily and discussed afterward.

\subsection{Virtual samples}

The study of virtual volumes of foam has been preferred to the study of voxel-based datasets obtained by micro-tomography technique. Indeed, the cost of this technique is not relevant and it is hardly applicable for large experimental-numerical studies. We decided to develop a virtual material initially homogeneous and constituted of heterogeneities localized randomly in the cube (Fig. 2a).

The interest of such virtual samples is to create samples inside which the variability can be easily controlled thanks to random functions. Indeed, the number, the size, the position of the heterogeneities and their material properties can easily be adjusted thanks to the software program developed for this study. Finally, the software program creates the virtual samples by defining some stacks of RGB-images which can be displayed in a 3-D view (Fig. 2a). The RGB value of each voxel is therefore related to a set of mechanical properties (density $\rho$, Young's modulus $E \ldots$..). The objective of this research work is to study the influence of the
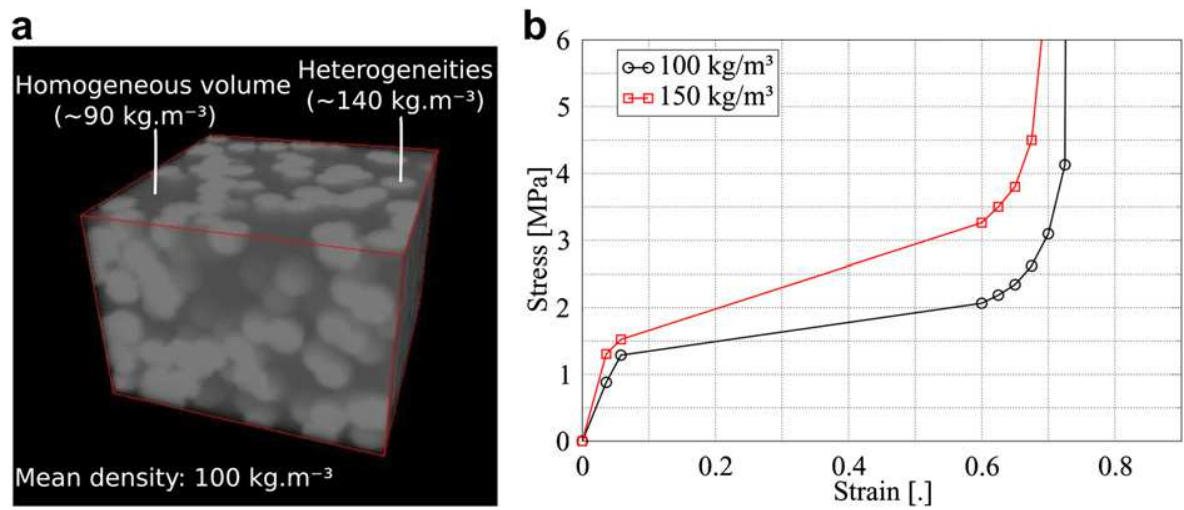

Fig. 2. Virtual sample of foam. (a) Description. (b) Dynamic behavior of foam with the density values of $100 \mathrm{~kg} \mathrm{~m}^{-3} \mathrm{and} 150 \mathrm{~kg} \mathrm{~m}^{-3}$ at a strain rate value of $200 \mathrm{~s}^{-1}$. 
a

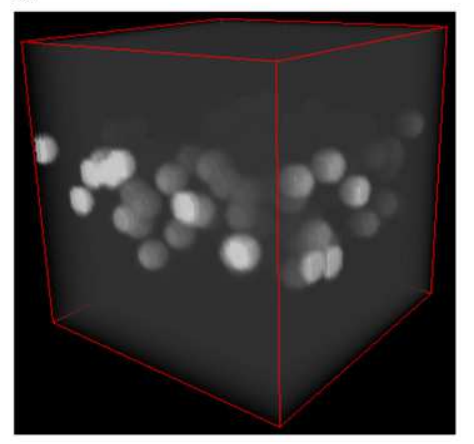

b

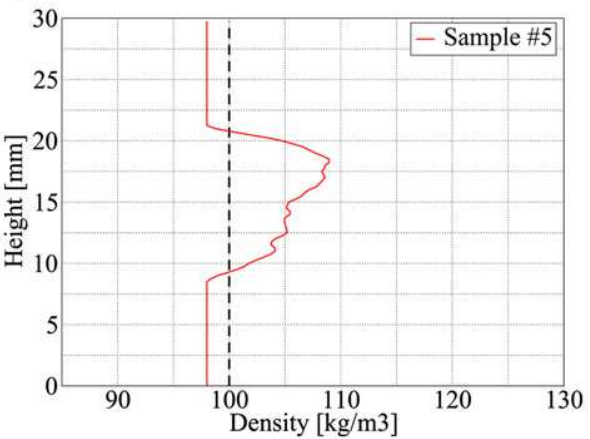

C

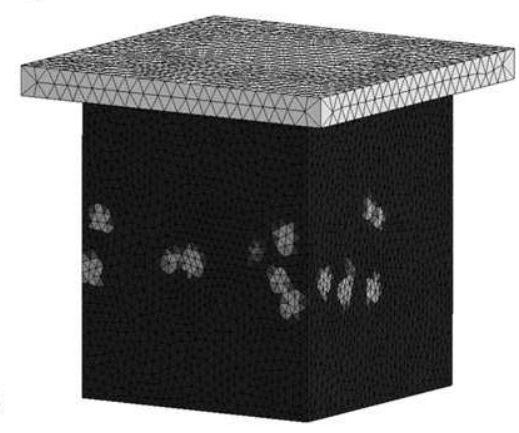

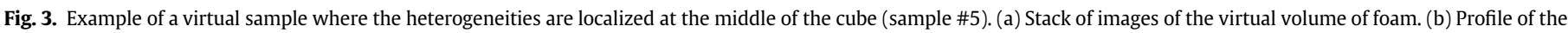
density range. (c) FE discretization.

Table 1

Characteristics of the virtual samples.

\begin{tabular}{lclll}
\hline Sample & Number & Size $[\mathrm{mm}]$ & Localization & $\begin{array}{l}\text { Volume ratio of } \\
\text { heterogeneities [\%] }\end{array}$ \\
\hline$\# 1$ & 337 & 3.6 & Randomly & 15.9 \\
$\# 2$ & 695 & 3.6 & Randomly & 29.4 \\
$\# 3$ & 354 & 7.2 & Randomly & 70.7 \\
$\# 4$ & 359 & 3.6 & Along a diagonal & 14.3 \\
$\# 5$ & 125 & 3.6 & Middle & $5.8(17.4$ locally) \\
$\# 6$ & 754 & 3.6 & Middle & $23.9(72.7$ locally) \\
$\# 7$ & 1747 & 3.6 & Middle & 48.3 (100 locally) \\
\hline
\end{tabular}

density variability and the grey level in the model is only for display purposes, representing the different densities.

Each virtual sample of foam can be characterized by its set of "number-size-position" of heterogeneities. However, even if these sets are useful to build different virtual volumes, they are not sufficiently explicit to explain the macroscopic results in this case of uni-axial compressive tests. Another way to characterize them is to plot a function of the value of density for each voxels' layer. This function gives more relevant characteristics to understand the macroscopic behavior of the foam sample. The Fig. 3a shows a virtual sample of foam where the 125 heterogeneities with a diameter of $3.6 \mathrm{~mm}$ are localized at the middle of the sample height. The characteristic function of this virtual sample is then plotted in the Fig. $3 \mathrm{~b}$.

The influence of the number, the size and the localization of the heterogeneities have been studied. To do so, several kinds of virtual samples have been build, three per kind of sample, to check repeatability and the representative samples are listed in the Table 1 . A difference in density of $50 \mathrm{~kg} \mathrm{~m}^{-3}$ has been arbitrarily applied between the heterogeneities and the rest of the volume with heavier heterogeneities and their behavior is clearly different (Fig. 2b). Viot et al. [7] have shown that this density range can reach $200 \mathrm{~kg} \mathrm{~m}^{-3}$ but the manufacturers try to reduce this variation in density in the microstructure to obtain more homogeneous material. Moreover, it is difficult to estimate precisely the density range in industrial foams. First simulations have also being performed by using a density range of $20 \mathrm{~kg} \mathrm{~m}^{-3}$ and, the results being similar, just the results of the simulations using $50 \mathrm{~kg} \mathrm{~m}^{-3}$ are presented in this paper. In order to compare virtual volumes with each other, their mean density has been globally adjusted to reach a value of $100 \mathrm{~kg} \mathrm{~m}^{-3}$.

In the Table 1, the ratio of the heterogeneities' volume over the total volume is also given. This variable is interesting to be considered in order to differentiate the samples with each other.

\subsection{FE simulation of dynamic compression of virtual samples}

The virtual samples of foam are then discretized into finite elements. The software program calculates the mean of RGB value of each voxel included in each finite element. This operation means to give different material properties up to 256, i.e. the number of RGB values, to each finite element. The grey level of the picture gives therefore a display of the density. A linear function of the density versus the RGB values has been chosen for this study. It is just a way to represent the variation of the density in the sample. The discretization into FE for a virtual sample of foam can be observed in Fig. 3c.

The calculations have been performed on the LS-Dyna software [12]. The behavior law used for this study is the *MAT_FU_ CHANG_FOAM [13] which describes the behavior of non-crushable
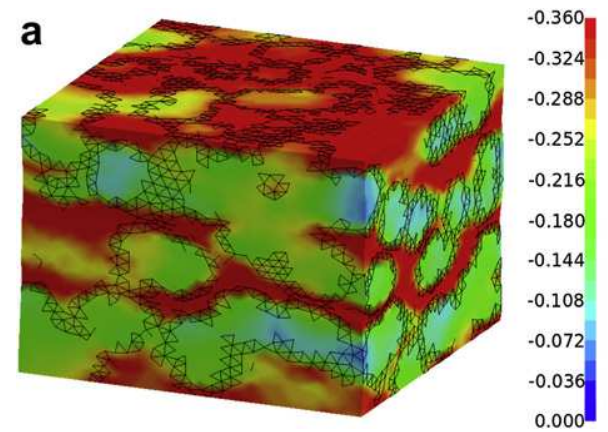

b

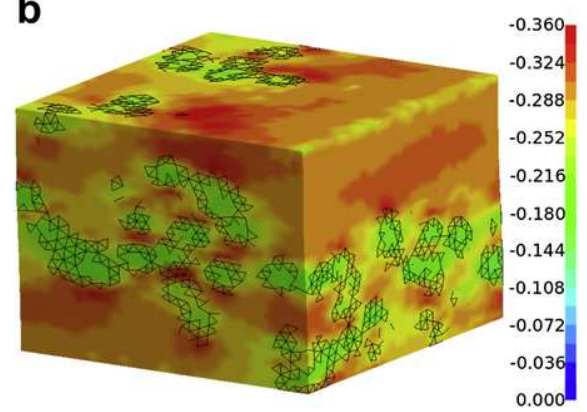

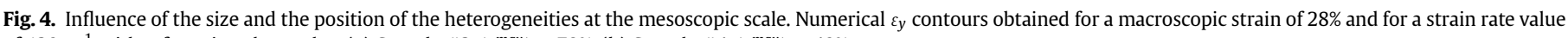
of $130 \mathrm{~s}^{-1}$ with a few virtual samples. (a) Sample \#3, $\left|\varepsilon_{y}^{\max }\right|=79 \%$. (b) Sample \#4, $\left|\varepsilon_{y}^{\max }\right|=40 \%$. 

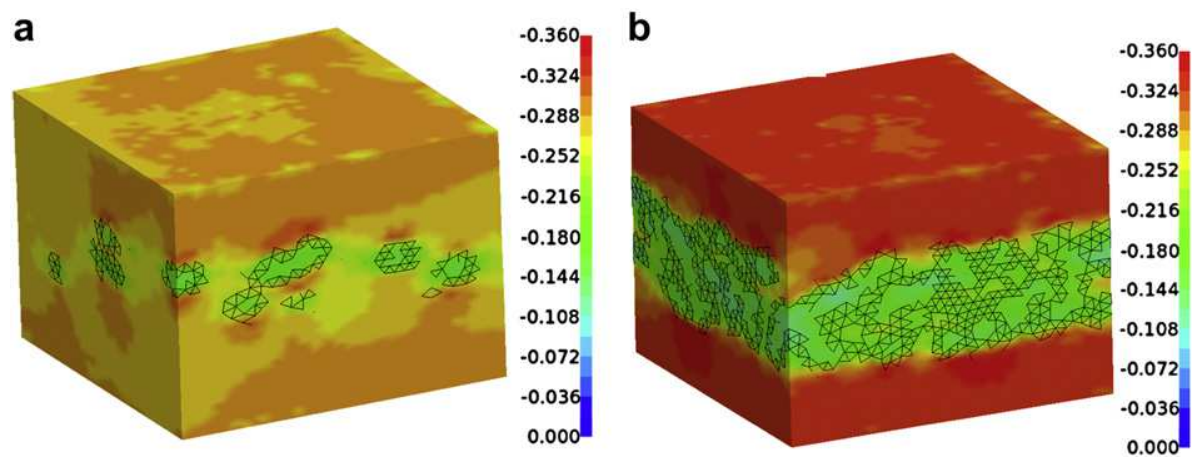

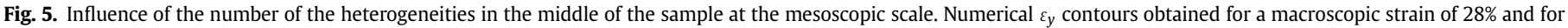
a strain rate value of $130 \mathrm{~s}^{-1}$ with a few virtual samples. (a) Sample \#5, $\left|\varepsilon_{y}^{\max }\right|=33 \%$. (b) Sample \#6, $\left|\varepsilon_{y}^{\max }\right|=41 \%$.

foams. As well as using basic parameters (i.e., the density and the Young modulus), this law needs data such as the strain-stress curves at different strain rates in order to model rate effects. The curves obtained by Bouix et al. [9] and described in Section 2.1 have been used for the current study.

The first numerical study deals with the uni-axial compression of virtual samples of foam. It can be expressed numerically by imposing a negative vertical displacement on the plane impactor. A velocity of $3.9 \mathrm{~m} \mathrm{~s}^{-1}$ with a maximum displacement of $21 \mathrm{~mm}$ was applied on the impactor. The nodes at the bottom of the cube were fixed from any negative axial displacement but transversal displacement was allowed. A classical penalty contact for soft materials was defined between the impactor and the cube. The cube was discretized by more than 160,000 linear tetrahedron elements. The choice of the finite elements is related to their capacity to overcome several numerical problems. The strain level of foam materials uses to reach more than $90 \%$ and the use of the finite element method shows therefore its limits. Indeed, negative volumes of finite elements involve the forced end of the calculation. One way to overcome this problem [12] is to use under-integrated linear hexahedric elements with a Hourglass control or to use fullyintegrated linear tetrahedron. The main advantage of the latter elements is their great capacity to describe (overtake) large volume change without using any Hourglass correction.

\subsubsection{Mesoscopic behavior}

At the mesoscopic scale, the numerical results were analyzed by using the contours of the axial strain along the vertical axis (Figs. 4, 5 and 6a). With all the virtual samples, the maximum strain is observed in the thinnest vertical spaces between two heterogeneities and the minimum coincides with the localization of the heterogeneities. However, some differences can be observed between virtual samples.

With the sample \#3 (Fig. 4a), i.e. with big heterogeneities, the vertical spaces between the heterogeneities are very small and the strain level can reach a maximum of $79 \%$ for a macroscopic strain of $28 \%$. Horizontal paths of localized strain can also be observed between heterogeneities. The strain level inside the heterogeneities is about $10 \%$ showing a very large range of strain inside the sample. With the sample \#4 (Fig. 4b), i.e. the heterogeneities are localized along a diagonal of the volume, the range is not as large as the previous sample and the maximum axial strain is about $40 \%$ for a macroscopic strain of $28 \%$. The strain is rather localized on every side of the diagonal but strain localization is hardly observable.

When the sample \#5 (Fig. 5a) is compared with the sample \#6 (Fig. 5b), it can be seen that higher volume of heterogeneities in the middle of the virtual volume caused more strain localization in the upper and lower parts of the sample. For a macroscopic strain of $28 \%$, the mean of the axial strain at the top and the bottom of the sample reaches approximately $29 \%$ and $33 \%$ with the samples \#5 and \#6, respectively. It shows that even if the volume ratio of heterogeneities is about $72 \%$ in the middle of the sample with a density difference of $50 \mathrm{~kg} \mathrm{~m}^{-3}$, the strain value in the localized zone is only about $5 \%$ more than the macroscopic strain.

The numerical results (sample \#1, Fig. 6a) can also be compared with the experimental data thanks to the Digital Image Correlation (DIC) technique used for the dynamic compressive load of a homogeneous sample of foam. The software CORRELI-Q4 has been used for the study as in [14]. The contours of the axial strain for a Zone Of Interest (ZOI) of $8 \times 8$ are displayed in the Fig. $6 \mathrm{~b}$. The cumulated error which comes from the DIC technique does not exceed $2.5 \%$. Localization of the strain can be observed in the face of
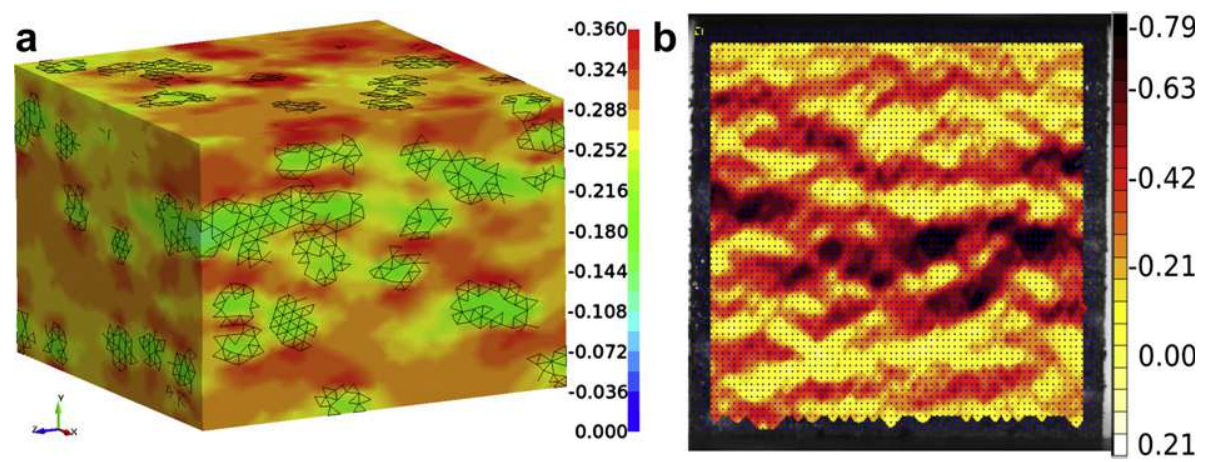

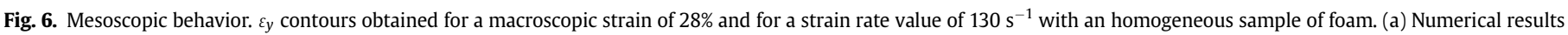
obtained with the FE simulation for the sample $\# 1,\left|\varepsilon_{y}^{\max }\right| w=36 \%$. (b) Experimental results obtained with the DIC technique, $\left|\varepsilon_{y}^{\max }\right|=79 \%$. Cumulated error $=2.5 \%$. 

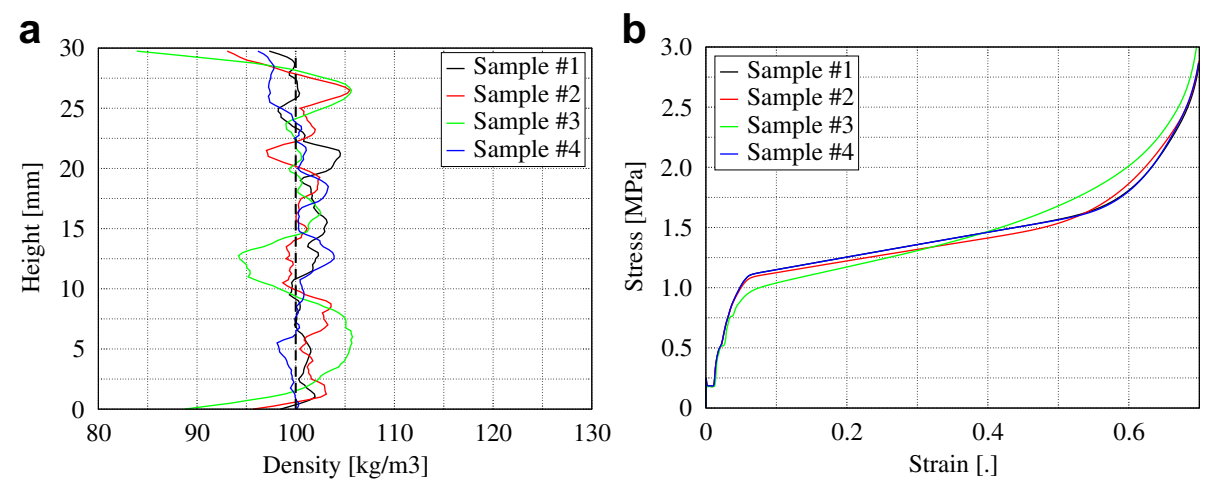

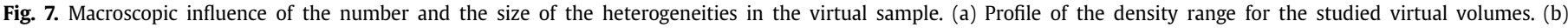
Stress-strain curves.

the sample. Experimentally, for this sample, the maximum of the axial strain reaches $79 \%$ for a mean strain of $28 \%$. The value is underestimated in the numerical sample \#1 (36\%) compared to experiments. Several reasons can explain this difference. First, this can be due to a too small density range, i.e. $50 \mathrm{~kg} \mathrm{~m}^{-3}$, with this kind of organized system of heterogeneities. In this case, the approximation of this range performed for another EPP material by Viot et al. [7] and mentioned previously (Section 2.1) would be a mean to reduce the difference. Further investigations related to this topic will be part of future research work. Second, another way to approach the DIC results is to modify the organized system of heterogeneities itself. Such strain localization can be observed in the numerical sample \#3 which shows that the volume ratio of heterogeneities must be sufficiently important to reach this strain level obtained experimentally.

\subsubsection{Macroscopic behavior}

The influence of the number, the size and the position of the heterogeneities on the macroscopic behavior is firstly studied.

Fig. 7a shows the variation of the density in the height of the virtual samples \#1, \#2, \#3 and \#4 is randomly centered on the mean value of $100 \mathrm{~kg} \mathrm{~m}^{-3}$. As mentioned previously, this function shows that samples created from different sets of "number-size" of heterogeneities can nearly involve the same repartition of the heterogeneities in the height of the sample. With foam samples constituted by a difference of $50 \mathrm{~kg} \mathrm{~m}^{-3}$ between the heterogeneities and the rest of the volume, the macroscopic behavior is nearly the same whatever the number and the size of the heterogeneities in the foam sample (Fig. 7b). However, one can note that the behavior of the sample \#3 is slightly different. This is due to the evolution of the density in the height of the sample. Its density range is about [83:106] $\mathrm{kg} \mathrm{m}^{-3}$ and the density difference in the height of the sample which is therefore about $23 \mathrm{~kg} \mathrm{~m}^{-3}$. This density range does not exceed 7,12 and $8 \mathrm{~kg} \mathrm{~m}^{-3}$ with the samples \#1, \#2 and \#4, respectively. These results show that the macroscopic behavior is affected by the mean of the mechanical properties in the height of the virtual foam.

A second step of calculations was performed to show the influence of the volume ratio of the heterogeneities when they are localized in the middle of the volume. The Fig. 8a shows the variation of the density range for the samples \#5, \#6 and \#7. The mean density is therefore locally modified in the middle of the virtual samples and the difference in density between the middle and the rest of the volume is about $10,30,50 \mathrm{~kg} \mathrm{~m}^{-3}$ for the samples \#5, \#6, \#7, respectively.

It can be seen in Fig. $8 \mathrm{~b}$ that the macroscopic behavior is only different when the volume ratio of heterogeneities in the middle of the volume is sufficiently important. The heterogeneous zones are no more at the mesoscopic scale but have been fused to the macroscopic scale and the virtual sample with mesoscopic heterogeneities can be now considered as a multi-layered volume of foam. Finally, the only way to modify the macroscopic behavior of foam in the loading case of uni-axial compressive tests is to pile up layers of different densities.

\section{Multi-layered foams}

The previous numerical study on the influence of the mesoscopic heterogeneities to modify the macroscopic behavior showed the potential of multi-layered foams in the case of compressive a

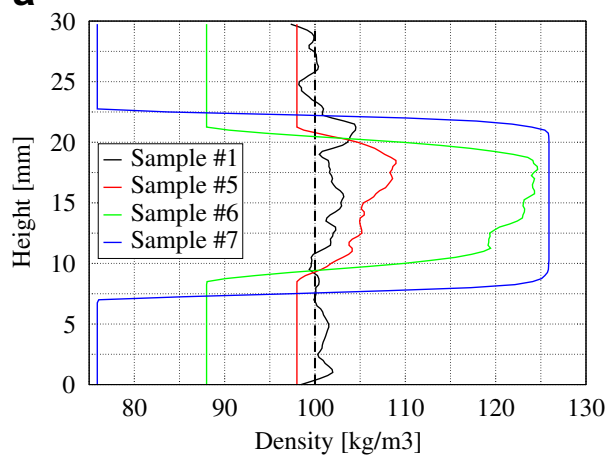

b

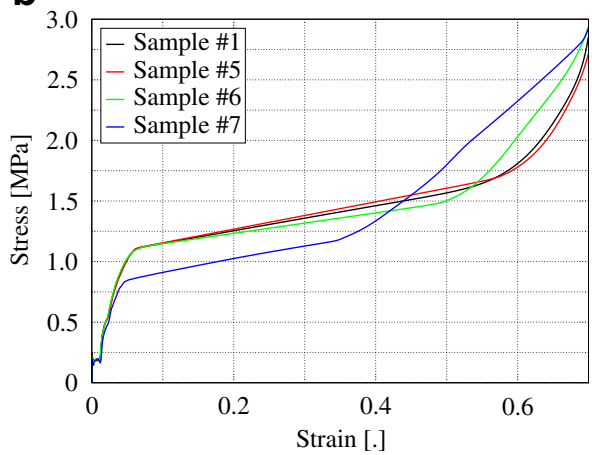

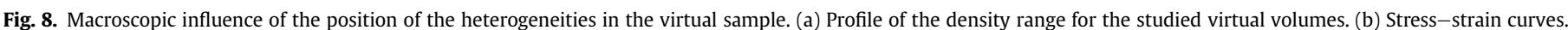


Table 2

Characteristics of the experimental samples of foam.

\begin{tabular}{lcccl}
\hline Sample & Densities & & & \\
\cline { 2 - 5 } & $\begin{array}{c}\text { Layer \#1 } \\
{\left[\mathrm{kg} \cdot \mathrm{m}^{-3}\right]}\end{array}$ & $\begin{array}{c}\text { Layer \#2 } \\
{\left[\mathrm{kg} \cdot \mathrm{m}^{-3}\right]}\end{array}$ & $\begin{array}{l}\text { Layer \#3 } \\
{\left[\mathrm{kg} \cdot \mathrm{m}^{-3}\right]}\end{array}$ & $\begin{array}{l}\text { Mean } \\
{\left[\mathrm{kg} \mathrm{m}^{-3}\right]}\end{array}$ \\
\hline Homogeneous & \multicolumn{1}{c}{-} & - & - & $94 \pm 2$ \\
$60-90-110$ & $64 \pm 3$ & $86 \pm 6$ & $112 \pm 5$ & $89 \pm 1$ \\
$110-40-110$ & $109 \pm 3$ & $42 \pm 3$ & $112 \pm 5$ & $89 \pm 1$ \\
$60-130-60$ & $62 \pm 2$ & $130 \pm 4$ & $67 \pm 2$ & $87 \pm 2$ \\
\hline
\end{tabular}

tests. One of the advantages of this conclusion was that it could be easily tested using experimental compressive tests. Indeed, foam layers can be assembled using neoprene glue to avoid any sliding. Only the results at the macroscopic scale will be discussed afterward because the mesoscopic results confirmed the results obtained previously.

\subsection{Experiments - quasistatic compressive tests}

One homogeneous and three different multi-layered foam samples with a size of approximately $30 \times 30 \times 30 \mathrm{~mm}^{3}$ were manufactured for this study and their characteristics are presented in the Table 2. The thickness of the three layers is equal to one third of the sample thickness. The experiments have been performed on five samples of each kind of foam in order to check the repeatability. In order to compare the results of each sample, the mean density of each one has been adjusted to approximately $90 \mathrm{~kg} \mathrm{~m}^{-3}$.

First, quasistatic compressive tests were performed on a Zwick electromechanical device at a strain rate of $0.01 \mathrm{~s}^{-1}$. The stress-strain curves of each sample are plotted in Fig. 9a. It can be seen that the macroscopic behavior of the multi-layered samples is different from each other and from the homogeneous one. The steps of the stress plateau can be easily observed for each value of density which constitutes the layer of the sample. For instance, the stress plateau of the $40 \mathrm{~kg} \mathrm{~m}^{-3}$ layer can be observed in the green curve at the beginning of the compressive test, i.e. when the strain is lower than 0.2. Following the steps of stress plateau and densification of the $40 \mathrm{~kg} \mathrm{~m}^{-3}$ layer, i.e. when the strain is in [0.3:0.6], the stress plateau of both $110 \mathrm{~kg} \mathrm{~m}^{-3}$ layers can be observed. One can also note that the length of the stress plateau is directly related to the thickness of its layer. Moreover, the order of the constituted layers does not have any influence on the macroscopic behavior in this case of compressive tests.

The strain localization can be observed in Fig. 10 and Fig. 11 for the samples 60-90-110 and 60-130-60, respectively. For each sample, five images are analyzed at five different strain values: 0 , $0.15,0.30,0.45$, and 0.60 . White lines have been displayed between each layer to appreciate the evolution of the layers thicknesses.

The Fig. 10 shows that even if the localization is mainly observed in the $60 \mathrm{~kg} \mathrm{~m}^{-3}$ layer, i.e. the bottom one, small strains appear progressively in other layers, $90 \mathrm{~kg} \mathrm{~m}^{-3}$ and $110 \mathrm{~kg} \mathrm{~m}^{-3}$. This is due to the small difference of density between the layers whereas in the 60-130-60 sample (Fig. 11), the strain level in the $130 \mathrm{~kg} \mathrm{~m}^{-3}$ middle layer is significantly less.

These different macroscopic behaviors involve different capacity to absorb energy. The absorbed energy versus the stress is plotted for each sample in the Fig. 9b and show the interest of multilayered foam to gradually absorb energy. When a homogeneous foam is tested, the foam does not absorb the energy before a stress of $0.5 \mathrm{MPa}$ whereas energy is absorbed from $0.2 \mathrm{MPa}$ with the ' $110-40-110$ ' sample. However, this latter foam is less efficient than others when the $40 \mathrm{~kg} \mathrm{~m}^{-3}$ layer reaches the step of densification, step during which a small quantity of energy is absorbed. This is due to the too large difference in density between the layers.
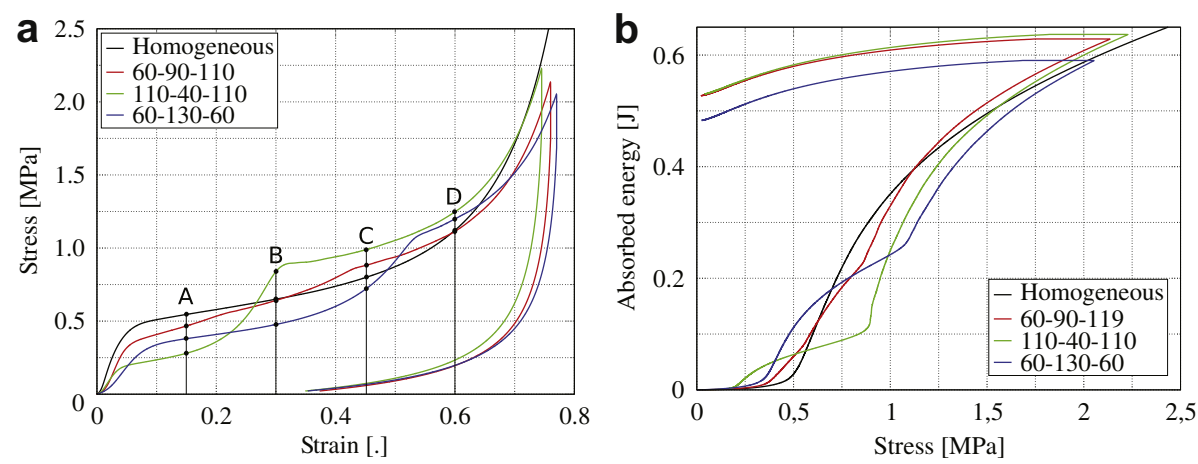

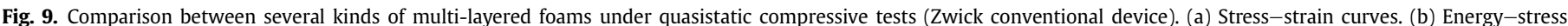
curves.
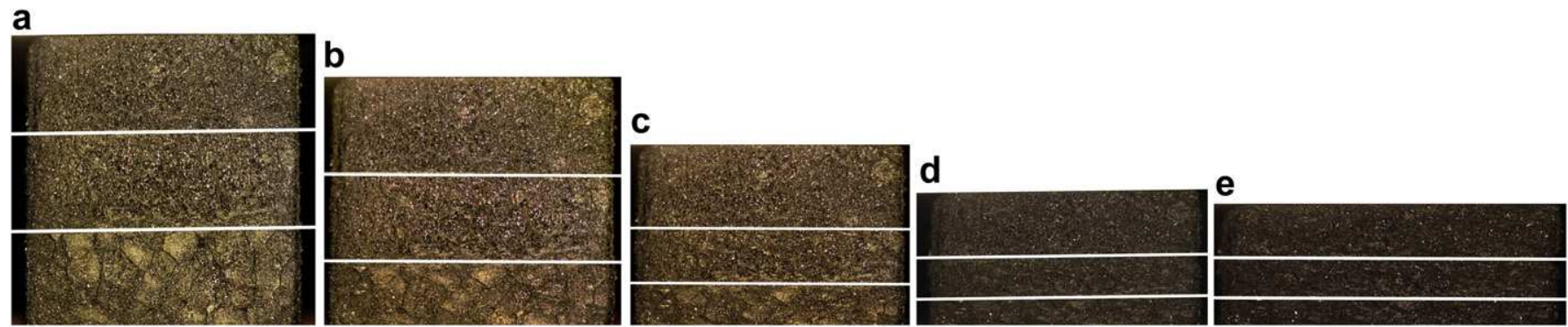

Fig. 10. 60-90-110 sample subjected to a quasistatic compressive test. (a) Initial. (b) A, $\varepsilon=15 \%$. (c) B, $\varepsilon=30 \%$. (d) C, $\varepsilon=45 \%$. (e) $D, \varepsilon=60 \%$. 
a
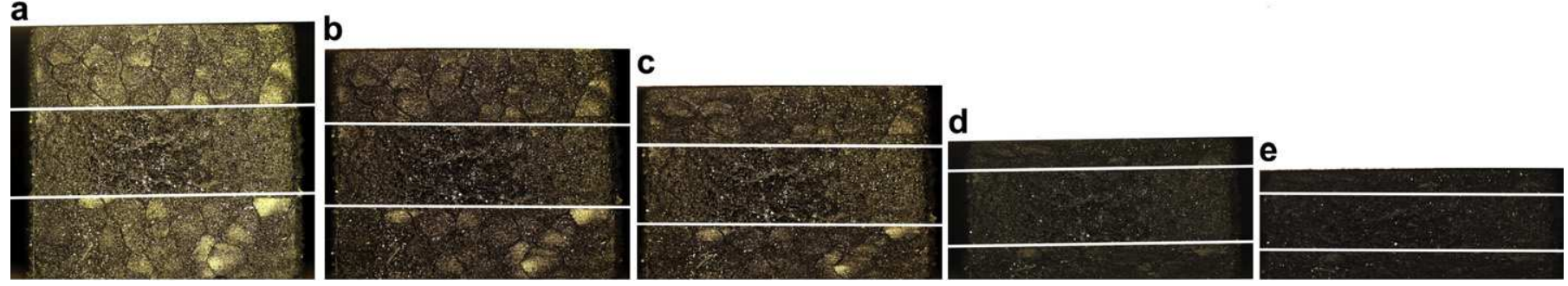

Fig. 11. 60-130-60 sample subjected to a quasistatic compressive test. (a) Initial. (b) A, $\varepsilon=15 \%$. (C) $B, \varepsilon=30 \%$. (d) C, $\varepsilon=45 \%$. (e) $D$, $\varepsilon=60 \%$.
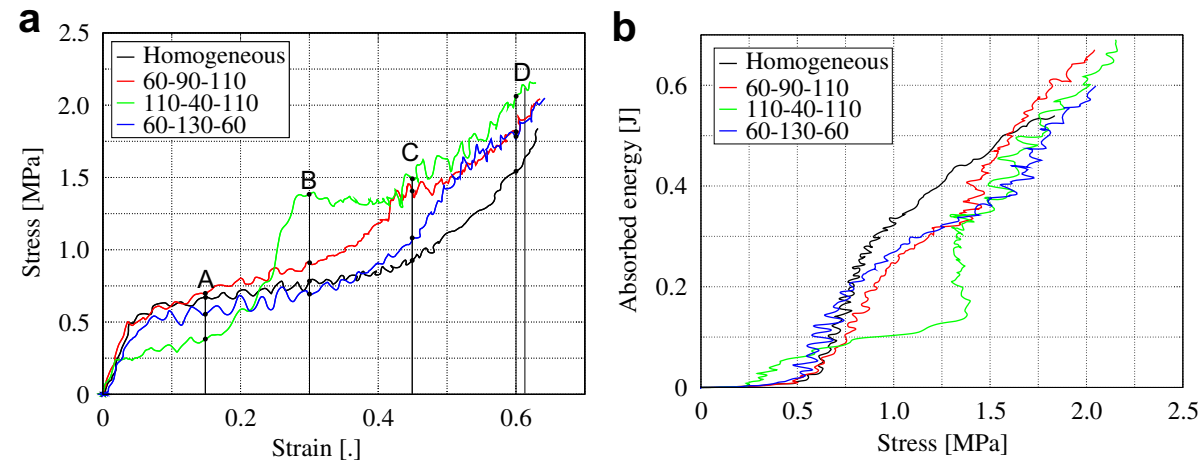

Fig. 12. Comparison between several kinds of multi-layered foams under dynamic compressive tests (Flywheel Device). (a) Stress-strain curves. (b) Energy-stress curves.

Indeed, the '60-90-110' sample (red curve) for which the density is gradually increased seems to be the most adapted to impacts for which a gradual energy absorption is required.

\subsection{Experiments - dynamic compressive tests}

Dynamic compressive tests have been performed with an original Flywheel device [9] which allows a quasi-constant strain rate during the test. The macroscopic strain rate chosen for this study is fixed to $110 \mathrm{~s}^{-1}$.

The stress-strain curves obtained with the dynamic compressive tests and observed in the Fig. 12a show similar results with the quasistatic tests. Nevertheless, oscillations due to wave reflection in the device assembly perturb the measured signal. An increase in the stress level can be noted compared with quasistatic tests. However, with the $94 \mathrm{~kg} \mathrm{~m}^{-3}$ homogeneous sample, the slight increase observed in the Fig. 12a is not consistent with the results obtained by Bouix et al. [9]. The results obtained with this foam has therefore to be carefully analyzed. The same trends of the absorbed energy in dynamics can be observed for the multi-layered samples (Fig. 12b) compared to the quasistatic tests. It can also be shown that gradually dense foams, such as the 60-90-110, allow a progressive absorption of the energy impact without involving an energy absorption plateau due to too large density difference between the layers.

The results of the dynamic compressive tests are also presented thanks to images obtained during the test (Figs. 13-15). One can see that the strain is first localized in the less dense layers of foam. However, with the same manner as in dynamic, the strain is progressively distributed in all the layers in the 60-90-110 sample (Fig. 13). Because of large differences in density between layers in the $110-40110$ (Fig. 14) and 60-130-60 (Fig. 15) samples, the strain is distributed in the most dense layers after a long step of densification for the least dense layers.

\subsection{Numerical simulation - dynamic compressive tests}

FE simulation of the dynamic compressive tests were performed using LS-Dyna software with the same input data as in the Section 2.3. The numerical results are presented in term of the stress-strain and the absorbed energy versus stress curves in the Fig. 16. The

a

b
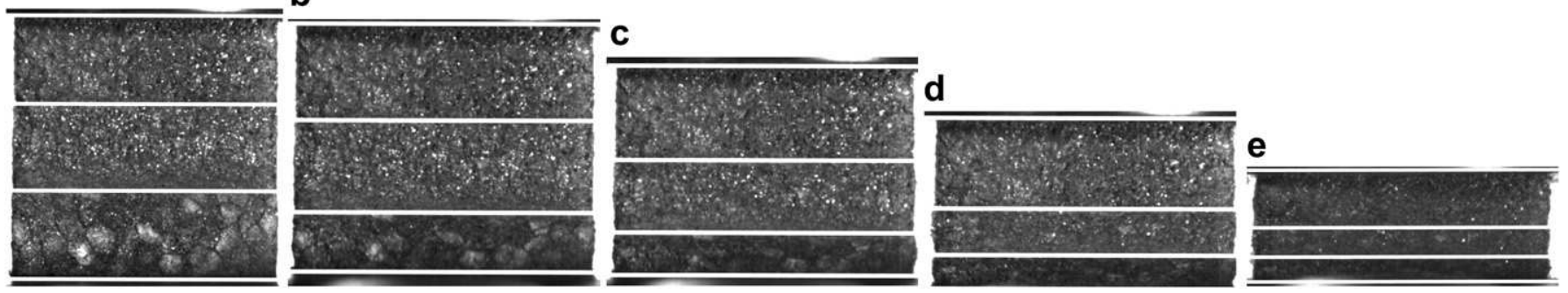

Fig. 13. 60-90-110 sample subjected to a dynamic compressive test. (a) Initial. (b) A, $\varepsilon=15 \%$. (C) $B, \varepsilon=30 \%$. (d) C, $\varepsilon=45 \%$. (e) $D, \varepsilon=60 \%$. 

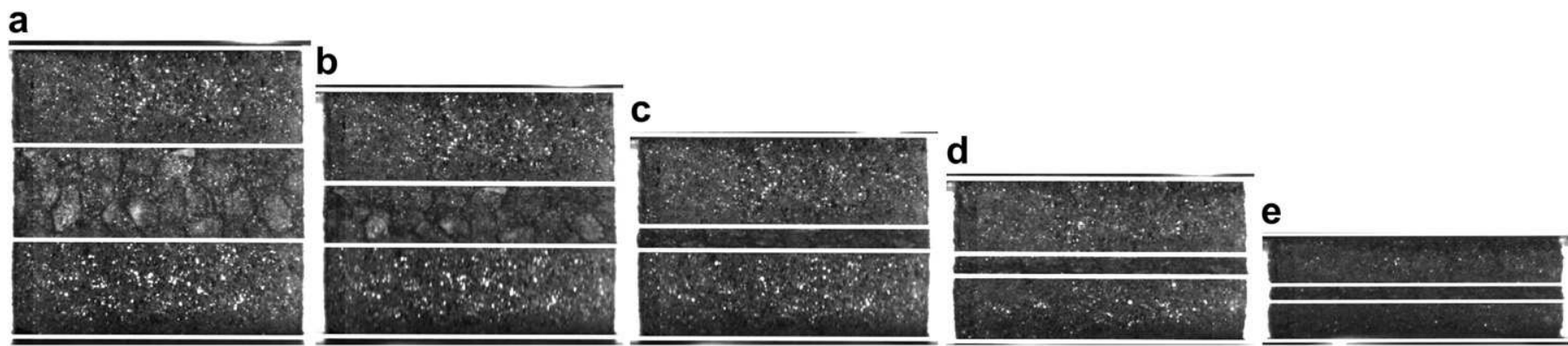

Fig. 14. 110-40-110 sample subjected to a dynamic compressive test. (a) Initial. (b) $A, \varepsilon=15 \%$. (C) $B, \varepsilon=30 \%$. (d) $C, \varepsilon=45 \%$. (e) $D, \varepsilon=60 \%$.

a
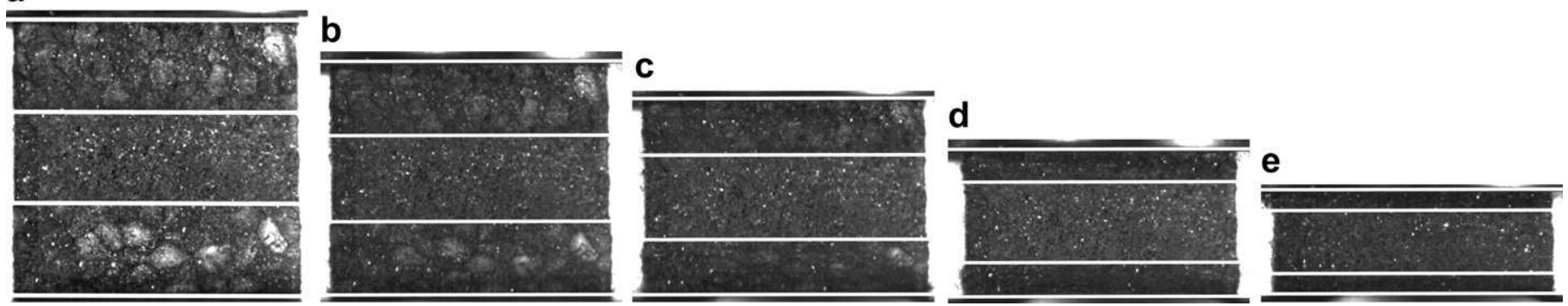

Fig. 15. 60-130-60 sample subjected to a dynamic compressive test. (a) Initial. (b) A, $\varepsilon=15 \%$. (C) B, $\varepsilon=30 \%$. (d) C, $\varepsilon=45 \%$. (e) D, $\varepsilon=60 \%$.
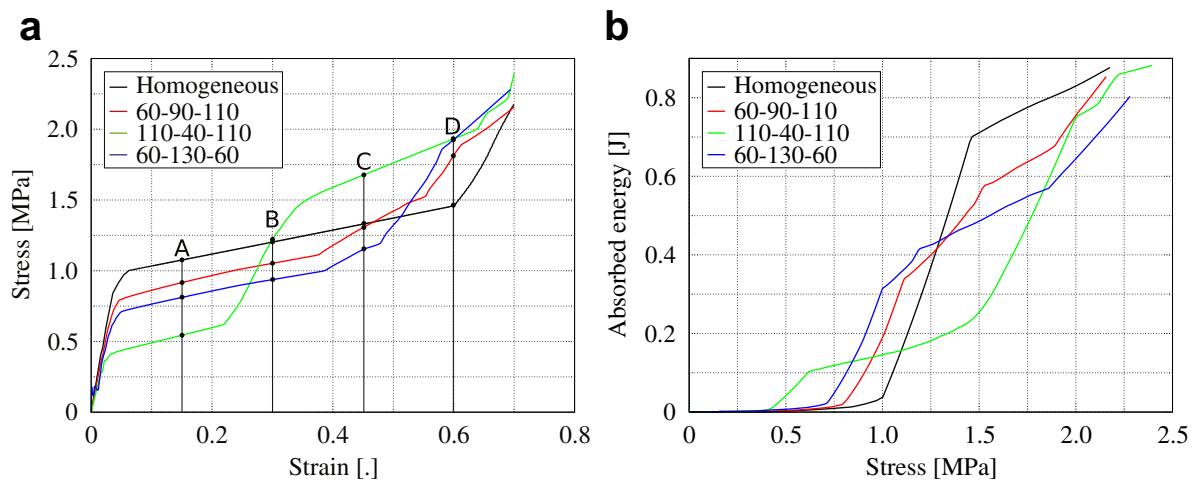

Fig. 16. Comparison between several kinds of multi-layered foams under dynamic compressive tests (Numerical results). (a) Stress-strain curves. (b) Energy-stress curves.

macroscopic behavior of each multi-layered sample is well described by the FE simulation (Fig. 16a) and one can easily see the different stress level of each layer. The evolution of the curves are not as smooth as with the experimental quasistatic tests and the 'straight line' shape of these curves can be explained by the limited number of recorded points, i.e. 15 points, used to describe the behavior. Even if the trends are the same with the experimental dynamic compressive tests, the stress level of the first step of the test, i.e. when the strain is approximately lower than 0.4, is overestimated by the FE simulation with all the samples. This difference comes from a numerical artefact which is due to the use of numerical damping in the LS-Dyna software to overcome negative volumes in the finite elements [12,13]. A numerical stiffness which is added to soft finite elements involves an increase of the stress level in the elastic and plateau stages. With this kind of multilayered foams, the use of this numerical stiffness is necessary to

\section{a}
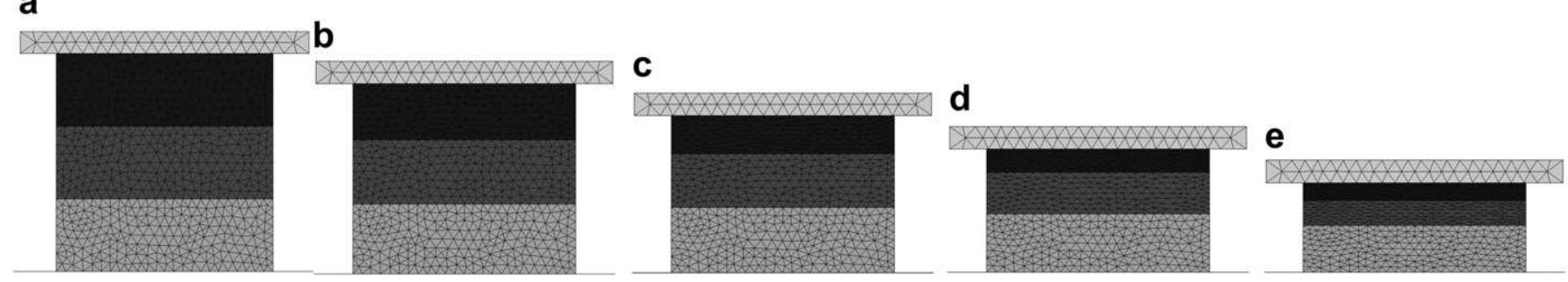

Fig. 17. Progression of compression in the 60-90-110 sample. (a) Initial. (b) A, $\varepsilon=15 \%$. (C) $B, \varepsilon=30 \%$. (d) C, $\varepsilon=45 \%$. (e) $D, \varepsilon=60 \%$. 
a

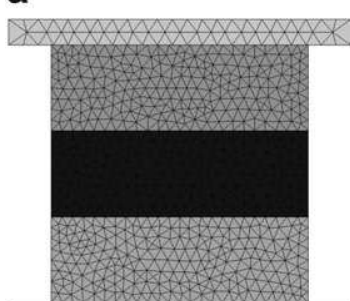

b

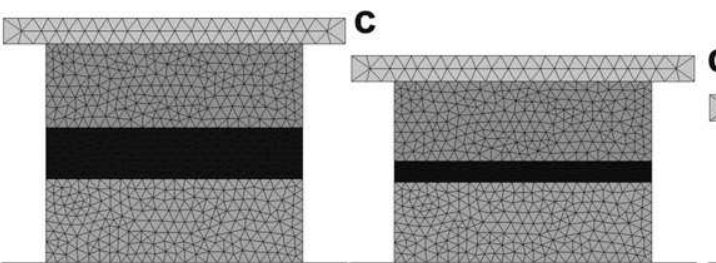

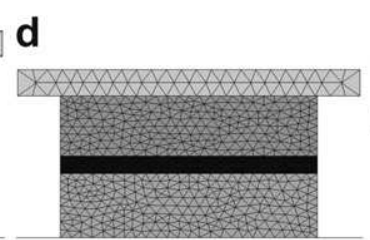

e

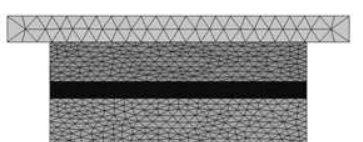

Fig. 18. Progression of compression in the $110-40-110$ sample. (a) Initial. (b) $A, \varepsilon=15 \%$. (C) $B, \varepsilon=30 \%$. (d) C, $\varepsilon=45 \%$. (e) $D, \varepsilon=60 \%$.

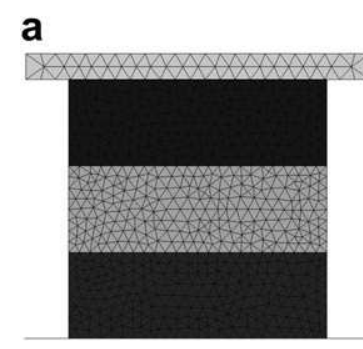

\section{b}
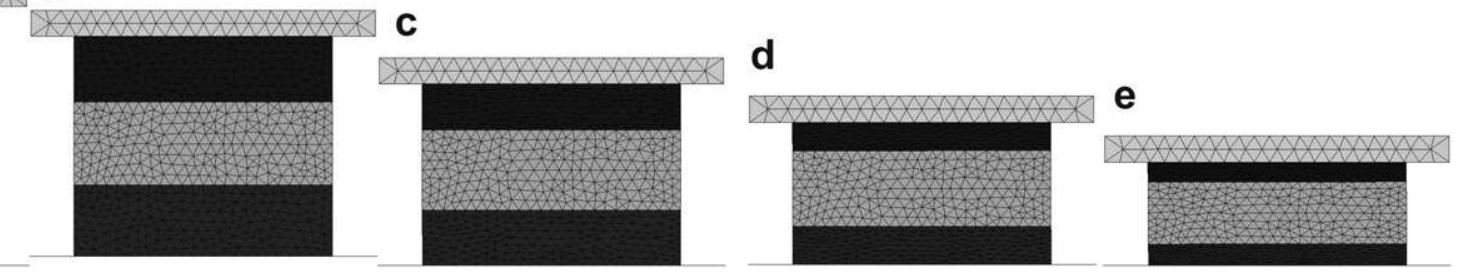

Fig. 19. Progression of compression in the 60-130-60 sample. (a) Initial. (b) A, $\varepsilon=15 \%$. (C) B, $\varepsilon=30 \%$. (d) C, $\varepsilon=45 \%$. (e) D, $\varepsilon=60 \%$.

prevent the soft layers from completely collapsing before the deformation of other layers.

The results of these numerical tests are also presented using images obtained during the test (Figs. 17-19). The trends are the same as with the experiments and show that the strain is localized firstly in the weakest layer of the samples. However, for a macroscopic strain of $45 \%$ (mark ' $C$ ' on the Figures), the strain is only localized in the weakest layer for the samples with a large density
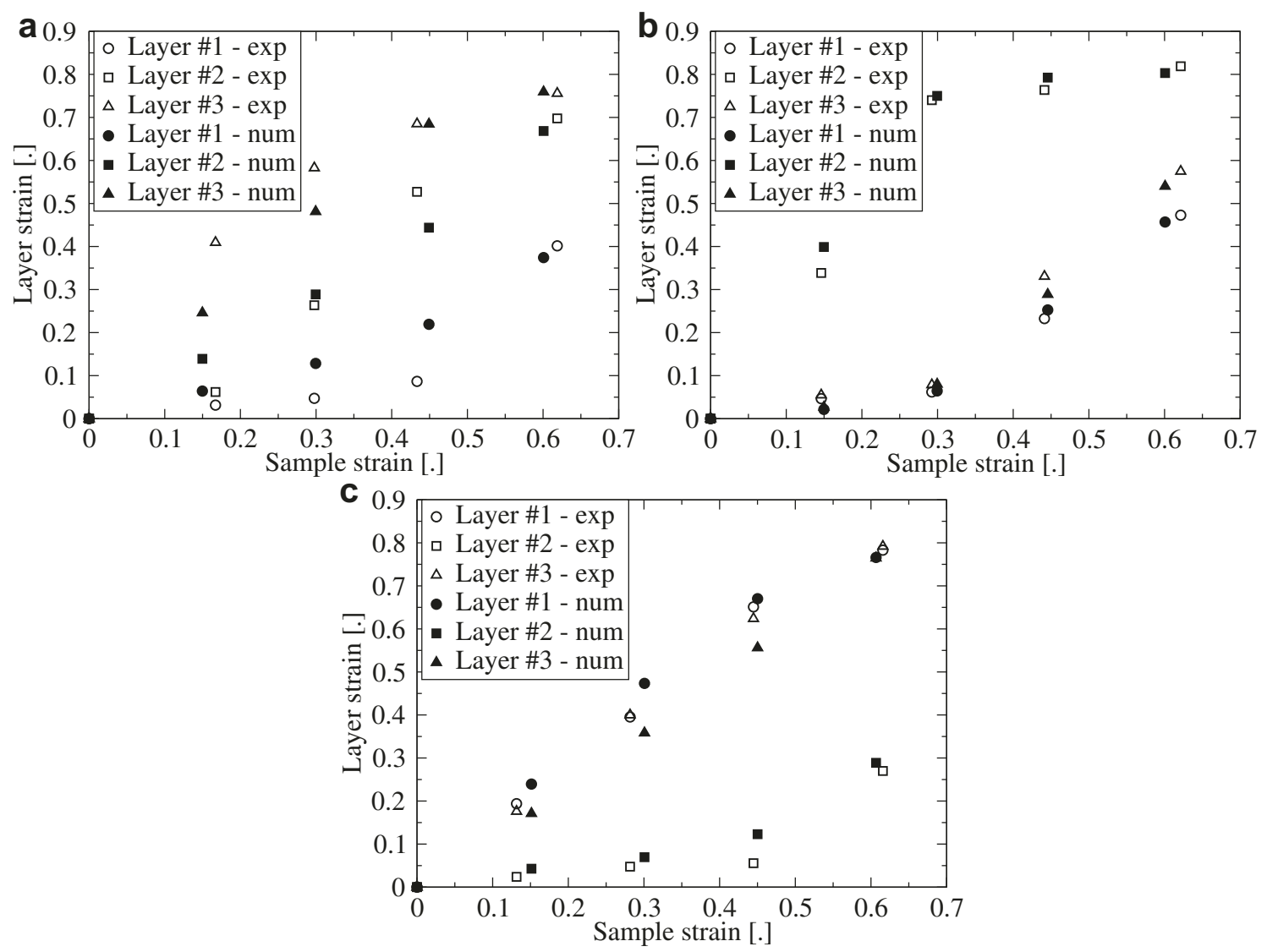

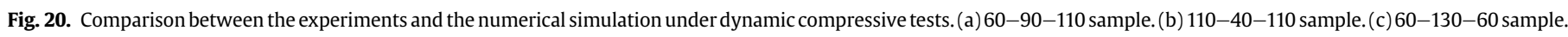


difference between layers (the 110-40-110 and the 60-130-60 samples) whereas the strain is distributed in the $60 \mathrm{~kg} \mathrm{~m}^{-3}$ and the $90 \mathrm{~kg} \mathrm{~m}^{-3}$.

For each layer of these three samples dynamically loaded, the strain of the layer can also be plotted versus the strain of the sample (Fig. 20). The strains of each layer of experimental samples are deduced from the variation of the thickness of each layer. This variation is directly measured on the images (Figs. 13-15). The strains of each numerical layer are obtained directly from the LSDyna software results.

It can be seen from the Fig. 20b and $c$ that there is a good agreement between numerical simulations and experiments. The difference of the strain level between numerical and experimental results does not exceed 0.1 in every layers and these results are acceptable considering the numerical assumptions. In the Fig. 20a, the difference between numerical and experimental results is higher than for the other cases (the difference between experimental and numerical strains are inferior to 0.16 for intermediate strain of the sample). However, this difference decreases for higher strain of the sample.

\section{Conclusion}

The polypropylene foam used for this study is defined as a multi-scale material which is constituted of mesoscopic beads. The large variability of the mechanical properties of the beads could therefore modify the macroscopic behavior. To study the influence of the beads variability on the macroscopic and local behaviors, virtual volumes of foam have been tested and compared in a Finite Element framework. Dense heterogeneities have therefore been introduced into the virtual volume and the effect of their size, number and place in the volume has been analyzed. It has been shown that in the case of a dynamic compressive test, a system of heterogeneities randomly placed in the volume modifies the mesoscopic behavior by the presence of strain localization but do not manage to change sufficiently the macroscopic behavior. The only way to modify it is to use multi-layered volumes of foam. The use of the Digital Image Correlation technique also allowed to discuss about the characteristics of the heterogeneities inside the volume.

A second part of this work dealt with the compressive tests of multi-layered volumes of foam to exhibit their energy absorption capacity. Experimental quasistatic and dynamic compressive tests have been performed and compared with the FE simulation results. Except for a small difference with the stress level, the FE simulation has good agreement with the experiments. This study has shown that a gradually dense foam allows a progressive absorption of the impact energy without involving a plateau due to too large density difference between the layers. This study enables a better understanding of cellular materials and will allow the designer to tailor the foam according to specification by controlling the response of the porous structure and to obtain optimal energy absorption.

\section{References}

[1] Mills N, Fitzgerald C, Gilchrist A, Verdejo R. Polymer foams for personal protection: cushions, shoes and helmets. Compos Sci Technol 2003;63(16): 2389-400. doi:10.1016/S0266-3538(03)00272-0.

[2] Gibson L, Ashby M. Cellular solids. Structure and properties. Cambridge Solid State Science Series. 2nd ed. Cambridge (UK): Cambridge University Press; 1997. pp. $175-234$.

[3] Forero-Rueda M, Cui L, Gilchrist M. Optimisation of energy absorting liner for equestrian helmets. Part i: layered foam liner. Mater Des 2009;30:3405-13. doi:10.1016/j.matdes.2009.03.037.

[4] Cui L, Forero-Rueda M, Gilchrist M. Optimisation of energy absorbing liner for equestrian helmets. Part ii: functionally graded foam liner. Mater Des 2009; 30:3414-9. doi:10.1016/j.matdes.2009.03.044.

[5] Cui L, Kiernan S, Gilchrist M. Designing the energy absorption capacity of functionally graded foam materials. Mater Sci Eng A 2009;507:215-25. doi: 10.1016/j.msea.2008.12.011.

[6] El-Hadek M, Tippur H. Dynamic fracture parameters and constraint effects in functionally graded syntactic epoxy foams. Int J Solids Structures 2003;40: 1885-906. doi:10.1016/S0020-7683(03)00028-3.

[7] Viot P, Plougonven E, Bernard D. Microtomography on polypropylene foam under dynamic loading: 3d analysis of bead morphology evolution. Compos A Appl Sci Manuf 2008;39(8):1266-81. doi:10.1016/j.compositesa.2007.11.014.

[8] Viot P, Beani F, Lataillade J-L. Polymeric foam behavior under dynamic compressive loading. J Mater Sci 2005;40:5829-37. doi:10.1007/s10853-005-4998-5.

[9] Bouix R, Viot P, Lataillade J-L. Polypropylene foam under dynamic loadings: strain rate, density and microstructure effects. Int J Imp Eng 2009;36(2): 329-42. doi:10.1016/j.ijimpeng.2007.11.007.

[10] Landi G, Niezgoda S, Kalidindi S. Multi-scale modeling of elastic response of three-dimensional voxel-based microstructure datasets using dft-based knowledge systems. Acta Mater 2010;58:2716-25. doi:10.1016/j.actamat. 2010.01.007.

[11] Viot P. Hydrostatic compression on polypropylene foam. Int J Imp Eng 2009; 36:975-89. doi:10.1016/j.ijimpeng.2008.11.010.

[12] Hallquist O. LS-Dyna keyword user's manual - version 971. Livermore Software Technology Corporation (LSTC); 2007.

[13] Hirth A, DuBois P, Weimar K. (Eds.), Improvement of LS-Dyna material law 83 (Fu Chang) for the industrial simulation of reversible energy-absorbing foams, CAD-FEM User Meeting, 1998.

[14] Roux S, Hild F, Viot P, Bernard D. Three-dimensional image correlation from Xray computed tomography of solid foam. Compos A Appl Sci Manuf 2008; 39(8):1253-65. doi:10.1016/j.compositesa.2007.11.011. 